SUBJECT AREAS:

TRANSMISSION ELECTRON MICROSCOPY

NANOSCALE MATERIALS

MATERIALS SCIENCE

Received

3 October 2014

Accepted

22 October 2014

Published

12 November 2014

Correspondence and requests for materials should be addressed to

C.O.K. lozsoy@is. mpg.de)

\section{Hybridization approach to in-line and off-axis (electron) holography for superior resolution and phase sensitivity}

\author{
C. Ozsoy-Keskinbora' ', C. B. Boothroyd ${ }^{2}$, R. E. Dunin-Borkowski², P. A. van Aken' \& C. T. Koch ${ }^{3}$
} 'Stuttgart Center for Electron Microscopy, Max Planck Institute for Intelligent Systems, Heisenbergstr. 3, 70569 Stuttgart, Germany,
2Ernst Ruska-Centre for Microscopy and Spectroscopy with Electrons and Peter Grünberg Institute, Forschungszentrum Jülich, 52425
Jülich, Germany, ${ }^{3}$ Institute for Experimental Physics, Ulm University, 89069 Ulm, Germany.

Holography - originally developed for correcting spherical aberration in transmission electron microscopes - is now used in a wide range of disciplines that involve the propagation of waves, including light optics, electron microscopy, acoustics and seismology. In electron microscopy, the two primary modes of holography are Gabor's original in-line setup and an off-axis approach that was developed subsequently. These two techniques are highly complementary, offering superior phase sensitivity at high and low spatial resolution, respectively. All previous investigations have focused on improving each method individually. Here, we show how the two approaches can be combined in a synergetic fashion to provide phase information with excellent sensitivity across all spatial frequencies, low noise and an efficient use of electron dose. The principle is also expected to be widely to applications of holography in light optics, X-ray optics, acoustics, ultra-sound, terahertz imaging, etc.

H olography was proposed by Denis Gabor in 1948 "to offer a way around" the resolution-limiting spherical aberration of the transmission electron microscope $(\mathrm{TEM})^{1}$. As a result of the development of the laser, the importance of holography as a technique for measuring both the amplitude and the phase of a wavefunction was soon realized and Denis Gabor was subsequently awarded the Nobel Prize in $1971^{2}$. In the TEM, holography is now used not only to correct microscope aberrations ${ }^{3}$ but also to characterize electrostatic potentials $^{4}$, charge order ${ }^{5}$, electric and magnetic fields ${ }^{6}$, strain distribution $s^{7,8}$, semiconductor dopant distributions $s^{9}$ and unstained biological specimens ${ }^{10}$, in each case with nanometer, sub-nanometer or even sub-Ångström spatial resolution. When examining biological or in general soft materials that contain primarily light elements, most structural information is carried in the phase of the elastically scattered wavefunction. However, such specimens are often beam-sensitive and require great care with regard to electron dose, as the ratio of inelastic (damaging) to elastic scattering events is high. It is therefore important to develop low-dose techniques for measuring the phase of electron wavefunctions quantitatively. Furthermore, investigating ordinary specimens over the full spatial frequency range with high resolution and high sensitivity is very challenging.

Holography, i.e., coherent wavefront reconstruction, can be performed using a wide variety of experimental setups. For electron holography alone, Cowley identified 20 independent form $\mathrm{s}^{11}$, of which the two most widely used modes are off-axis and in-line electron holography. Off-axis electron holography was pioneered by Möllenstedt and Düker ${ }^{12}$ and is based on the use of an electrostatic biprism, which usually takes the form of a charged wire placed in the electron beam path, as illustrated in Fig. 1a. The biprism is used to produce a fine interference fringe pattern, from which the complex wavefunction of the fast electron can be reconstructed using either linear algebra ${ }^{13}$ or an optical bench ${ }^{14}$. In-line electron holography (see illustration in Fig. $1 \mathrm{~b}$ ) ${ }^{1,15}$, which is also referred as focal series reconstruction, also works at much lower degrees of spatial coherence than off-axis electron holography, but requires the use of a computational algorithm to solve a non-linear ${ }^{16-18}$ (or, in some cases, approximated linear ${ }^{19,20}$ ) set of equations. These equations relate the complex electron wavefunction $\Psi(\boldsymbol{r})$ to image intensities $I(\boldsymbol{r}, \Delta f)$ that are usually recorded at multiple planes of focus, each characterized by its defocus $\Delta f$ from the reference focus at which the wavefunction is to be recovered. Off-axis electron holography has good phase sensitivity at low spatial frequencies, whereas either a large defocus range ${ }^{21,22}$, or variable defocus steps (as recently published by Haigh et $\mathrm{al}^{23}$ ), or model-based approaches $\mathrm{s}^{24,25}$ must be used to approach faithful reconstruction of low spatial frequency phase information by using in-line electron holography. At high spatial frequencies, both the spatial resolution and the phase sensitivity of in-line electron holography are higher than those of its off-axis counterpart for the same field of view and electron dose ${ }^{26}$. 

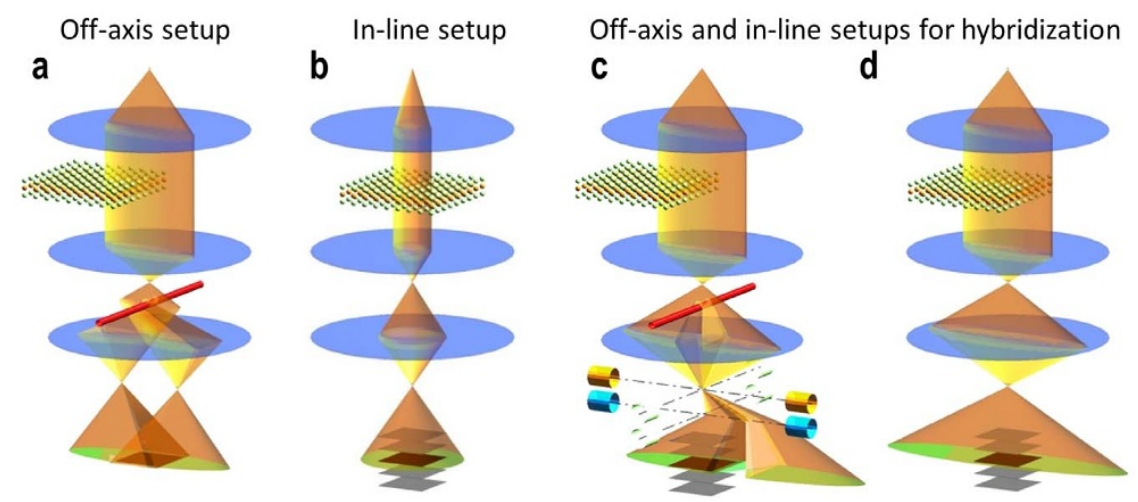

Figure 1 Schematic view of microscope setups: (a) off-axis and (b) in-line electron holography.(c) Shows how the in-line data can be acquired immediately after switching off the biprism, in a fully automated fashion by simply shifting the image. (d) Shows how the data have been acquired for the present work: the biprism was retracted, and the sample was slightly shifted to allow investigation of identical specimen areas by both methods.

Off-axis and in-line electron holography require very different optical setups for optimum performance and are highly complement$\operatorname{ary}^{10,22,26,27}$. For maximum phase sensitivity, off-axis electron holography is typically performed with highly elliptical illumination (Fig. 1a) (ellipticity ratios of $\varepsilon=30$ are common), whereas in-line electron holography is usually performed with isotropic spatial coherence (Fig. 1b). While high-frequency phase information is encoded very efficiently in in-line electron hologram ${ }^{26}$, experiments that aim at the reconstruction of low spatial frequency phase information are much more reliable (and quantitative even when using a low electron dose) when carried out using off-axis electron holography. Because of its need for highly coherent illumination, electron dose rates in offaxis electron holography are typically low and exposure times are very similar to the total exposure time of a focal series acquired for in-line electron holography. These fundamental differences between the two methods result from how phase information is encoded. While the off-axis setup encodes all spatial frequencies with equal strength (with a decrease in signal to noise ratio at higher frequencies), the phase sensitivity of in-line electron holograms is proportional to the spatial frequency squared $\left(\sim|\mathbf{q}|^{2}\right)$, i.e., it is low across long distances and higher for very fine details. For applications such as the measurement of magnetic fields, dopant distributions or concentrations of oxygen vacancies in oxides ${ }^{28}$, good phase sensitivity over the full range of spatial frequencies is required.

Here, an experimentally simple approach that combines in-line and off-axis electron holography and takes full advantage of their complementarity is presented, allowing a phase signal to be obtained with excellent signal-to-noise properties over all spatial frequencies. It also serves as a model for other holography applications at X-ray, microwave, radio, ultraviolet, visible optical wavelength $s^{29-35}$ etc., where shortcomings like the ones described above are observed due to the usage of either the in-line or the off-axis setup. The performance of the method is demonstrated by the investigation of ironfilled multi-walled carbon nano-onions. Off-axis and in-line electron holography experiments are carried out on the same region of the same sample with the same illumination conditions, allowing profiles of the projected electrostatic potential across individual particles to be determined quantitatively.

\section{Results}

In order to allow experimental conditions optimized for off-axis electron holography to be applied also for the in-line electron holography experiment, several approaches are possible, two of which are illustrated in Figs. 1c and 1d. In Fig. 1c the electrostatic biprism is left within the path of the electron beam, but the image shift coils are used to shift the area of the sample that has previously been investigated by off-axis holography on the detector. Since the image shift required for this is directly proportional to the biprism voltage that has been applied for the off-axis experiment, this procedure can easily be implemented in a fully automated fashion. For the proof-of-principle experiments presented below we have first removed the biprism, and then mechanically shifted the specimen, so that the area of interest was again visible on the detector (Fig. 1d). Since our simulations have shown that the relative benefit of combining in-line and off-axis electron holography is independent of the illumination ellipticity (see Fig. $4 \mathrm{i}-\mathrm{k}$ ), we set $\varepsilon=1$ in the experiments presented below.

Figure 2 shows an outline of the algorithm, illustrating the deterministic nature of linear off-axis electron holography reconstruction and the iterative nature of the refinement algorithm employed for inline electron holography reconstruction. It is a general feature of most iterative in-line electron holography reconstruction algorithms that the very strongly encoded high-frequency details of the phase of the wavefunction are reconstructed first, before slowly varying features in the phase are recovered ${ }^{29}$. In the presence of noise and residual inelastic scattering contributions to the experimental intensity measurements, the latter features may not be recovered at all. Fortunately, the refinement of experimental parameters and image registration is most sensitive to the accuracy with which high-frequency details have been estimated. It is therefore possible to first refine these details from a set of defocused images, using an empty phase map as a starting guess. During this process, the focal series can be aligned and the experimental parameters can be refined. The resulting estimate of the wavefunction can then simply be replaced with that recovered from an off-axis electron hologram and further refined by making it consistent with all in-line electron holograms. More specifically, the complete unwrapped phase and amplitude are imported separately, without applying any filtering for a specific frequency range. Since the iterative in-line reconstruction algorithm ensures that both the phase and the amplitude are consistent with the images in the focal series, the phase signal at low spatial frequencies is not affected significantly if only a few iterations are performed ${ }^{36}$. This procedure effectively extends the spatial resolution of the wavefunction obtained using off-axis electron holography, improves its signal to noise and removes the Fresnel fringes originating from the edges of the biprism.

Figure 3 illustrates results obtained by applying both off-axis and in-line electron holography alone and the combined (hybrid) approach to a sample of iron-filled multi-walled carbon nano-onions. The noise level in the wavefunction obtained using off-axis electron holography alone, which is much higher in both amplitude and phase (Figs. 2a-c) than that obtained using either the in-line or the hybrid method, results in part from the fact that highly elliptical illumination (which is normally employed for off-axis electron holography but has only a very subtle effect on the signal-to-noise properties of in-line 




Figure $2 \mid$ Schematic outline of the wave reconstruction algorithm used in the present work.

holograms) was not used in the present study. The application of in-line electron holography alone can be seen to reconstruct the amplitude well. However, ringing artifacts, which are not present in the off-axis reconstruction, are visible near sharp edges in the phase of the wavefunction (Figs. $3 \mathrm{~d}, \mathrm{f}$ ). The missing low spatial frequencies when using in-line electron holography can also be seen in a power spectrum generated from the reconstructed wavefunction (see below) ${ }^{37,38}$. Figure 3 shows that, when the two methods are combined, the spatial frequencies that are missing from the in-line electron holography result are recovered, while the higher resolution of the in-line approach is retained. The amplitude image is also improved, including the elimination of biprism fringes inherent in the off-axis technique. These results are also supported by the reconstructions from simulated data shown in Figure 4 . Due to the ability to directly compare the reconstructed phase images with the phase put into the simulations, the effectiveness of the hybrid approach can be verified in a very quantitative manner (see also Figs. 4i, 4k, and 4l). These simulations also allowed us to keep the electron dose exactly the same for all three techniques and easily test for the effect of changing the ellipticity of the illumination and verify that the hybrid approach presented here works very well at any (experimentally realizable) value of $\varepsilon$. Moreover, it clearly shows that the signal-to-noise properties of the phase image recovered by the hybrid approach are superior to those for both off-axis and in-line holography, individually, assuming exactly the same electron dose in all three cases.

Moving now back to the experimental data, Figure 5 shows power spectra generated from the three sets of experimental results, which illustrate the deficiency in information transfer in the phase obtained using in-line electron holography (Fig. 5a) up to a spatial frequency of $\sim 0.1 \mathrm{~nm}^{-1}$. Figure $5 \mathrm{~b}$ shows that the amplitudes reconstructed using the in-line and hybrid methods are much less noisy than those reconstructed using off-axis electron holography (see also Supplementary Fig. 1). The limited spatial resolution in the off-axis reconstruction is a result of an effective scattering angle limiting aperture applied during reconstruction. Using round, instead of elliptical illumination, the noise of the off-axis reconstruction is very high, despite an exposure time of $10 \mathrm{~s}$. The experimental data confirm our simulations in that at lower spatial frequencies the reconstructed phase is much more reliable if it is obtained using off-axis electron holography, whereas at higher spatial frequencies in-line electron holography provides the same information but with much less noise. The power spectrum of the phase obtained using the hybrid method shows a good match to that from the off-axis reconstruction at lower spatial frequencies (up to $\sim 0.2 \mathrm{~nm}^{-1}$ ), while above this frequency it converges to the power spectrum obtained using inline electron holography (Fig. 5 a,k).

Power spectra generated from the complex wavefunction (Figs. 5 d-f) demonstrate that, for the same field of view, the spatial resolution obtained using the in-line and hybrid methods is much better than that achieved using off-axis electron holography. In the example shown here, the cut-off resolution (outlined by a dashed red circle) was set to $0.405 \mathrm{~nm}$ for the in-line and hybrid methods. This is slightly larger than the physical objective aperture, whose shadow (outlined by a dashed yellow circle) can be seen in Figures $5 \mathrm{e}$ and $5 \mathrm{f}$. For the off-axis method, the resolution had to be limited to $0.972 \mathrm{~nm}$ 

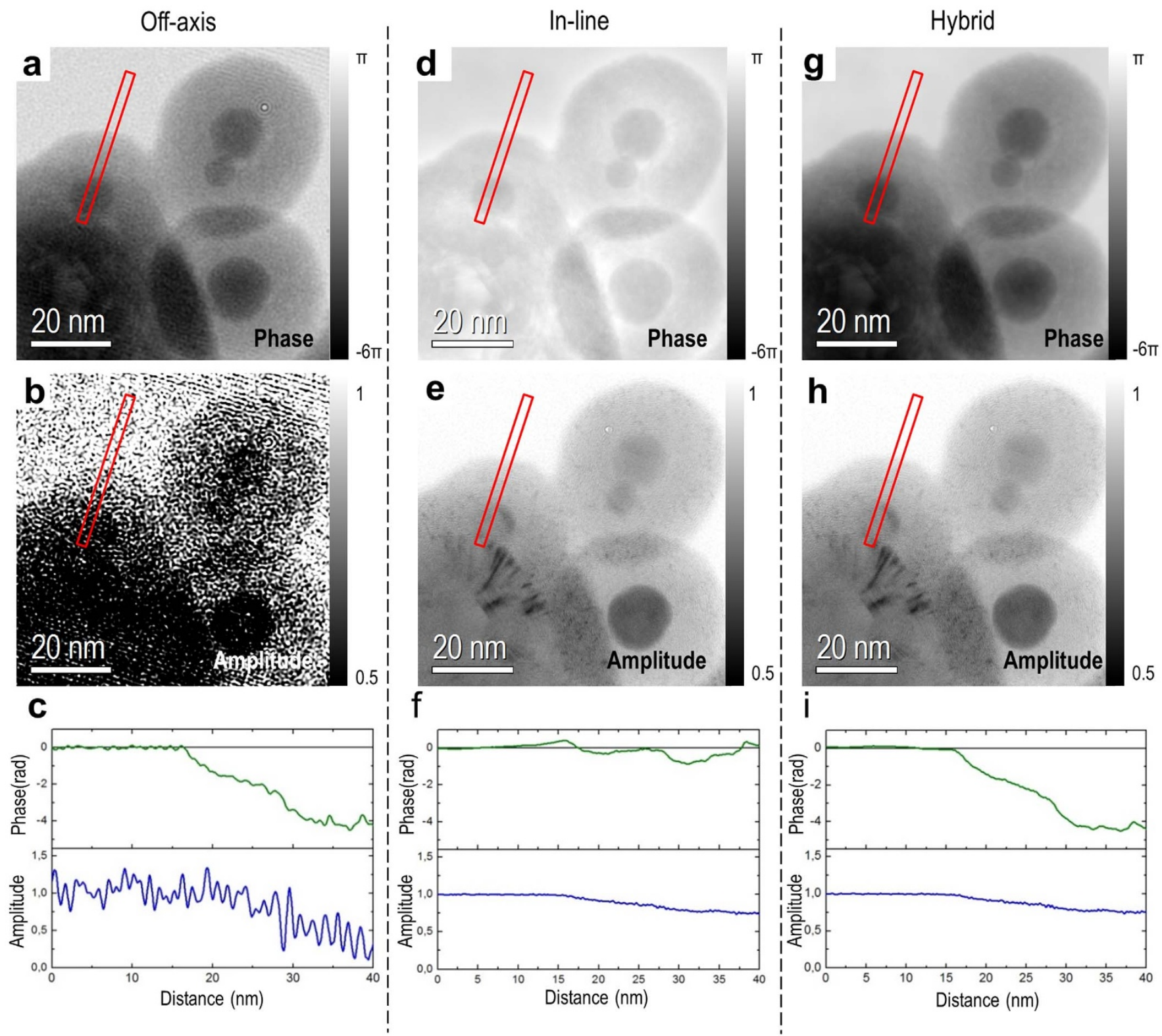

Figure 3 Comparison of results obtained from a sample of iron-filled multi-walled carbon nano-onions using three methods: top row (a, $d$, g) amplitudes; middle row (b, e, h) phases; bottom row (c, f, i) amplitude and background-subtracted phase profiles; left column (a, b, c) off-axis electron holography method; middle column (d, e, f) in-line electron holography method; right column (g, h, i) hybrid holography method.

by the numerical aperture size used during reconstruction. In addition to power spectrum analysis, profiles extracted from the diffractogram also support the information loss in the in-line electron holography phase image, and how much of this has been recovered by the hybrid method (Figs. $5 \mathrm{~g}$ and $5 \mathrm{k}$ ). Although magnification and thus the absolute aperture sizes can be increased, this will also cause an increase in noise, and the resolution ratio of the two techniques will remain similar if the same number of detector pixels is used for both methods.

\section{Discussion}

There are two main advantages of combining the in-line and off-axis methods: an increase in spatial resolution and a decrease in noise over the full range of spatial frequencies. For off-axis electron holography, the interference fringe spacing limits the maximum numerical aperture size that can be chosen during reconstruction. For a given illumination ellipticity, source brightness and exposure time, the fringe spacing cannot be decreased without increasing the noise.
For the in-line and hybrid methods, the physical objective aperture size can be increased up to the information limit. In Figure 6 and Table 1, a comparison of the reconstruction methods is presented for different frequency ranges of the phase images. A similar comparison is shown in Supplementary Fig. 1 for the amplitude images. In Figure 6, phase images filtered over three different spatial frequency ranges are shown for each of the three techniques, alongside profiles taken from areas marked by red boxes. The profiles illustrate the fact that the hybrid method matches the off-axis result at lower spatial frequencies (Figs. $6 \mathrm{a}-\mathrm{d}$ ) and the in-line result at higher spatial frequencies (Figs. $6 \mathrm{e}-\mathrm{h}$ ) and all show a good match in the intermediate frequency range (Figs. 6 i-1). From Table 1, it is apparent that for full and medium spatial frequency ranges $\left(0.1-1 \mathrm{~nm}^{-1}\right)$ the noise in the phase, estimated in the vacuum region where we expect the true phase to be flat, is approximately 4 times lower when using the hybrid method than for off-axis electron holography. The approximately 4 times lower noise in the phase, presented in Table 1, confirms that the hybrid method has better noise properties than off-axis electron 
Original image
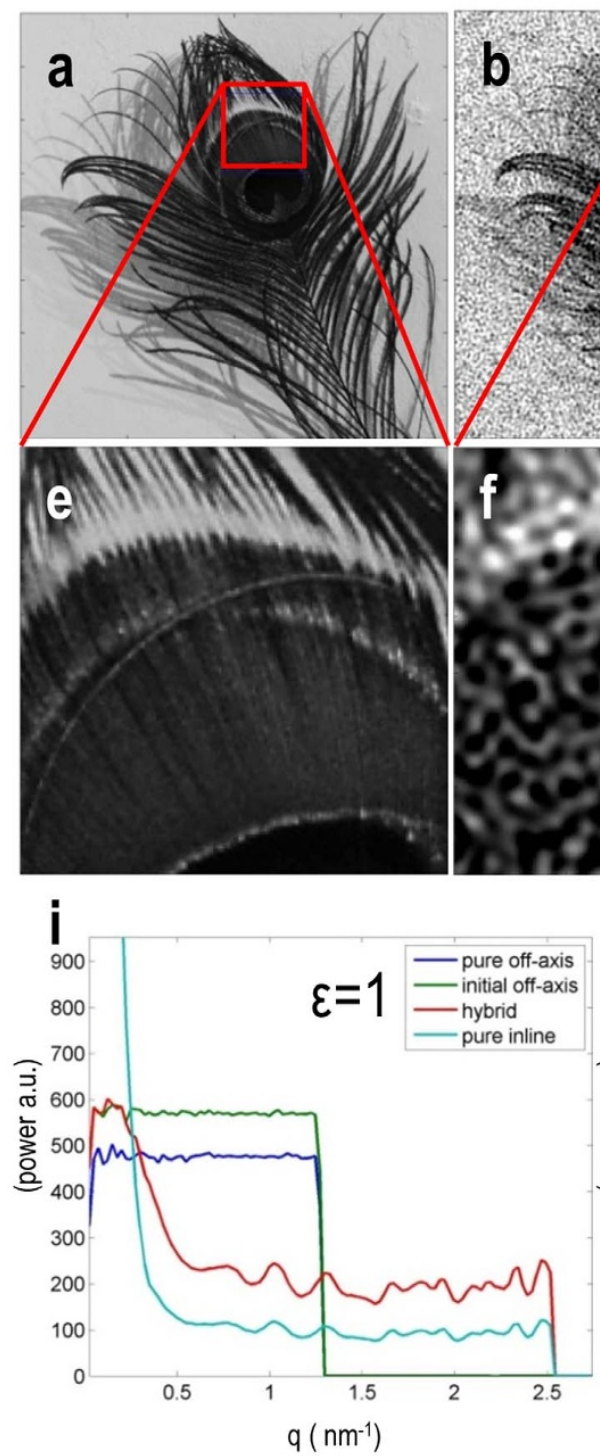

Off-axis image

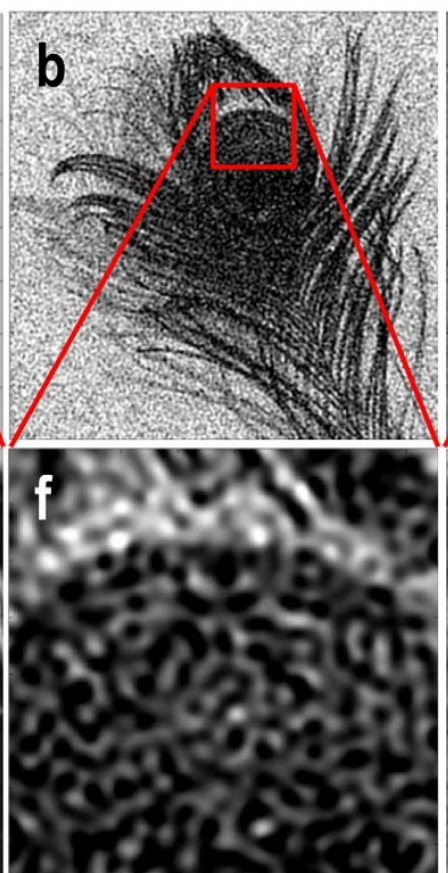

$\mathbf{k}$

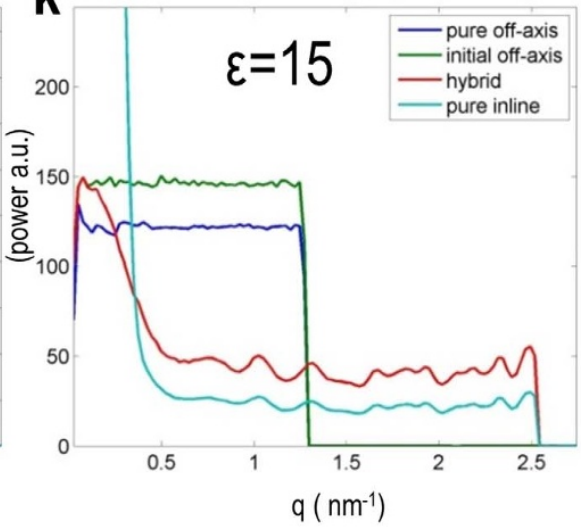

In-line image

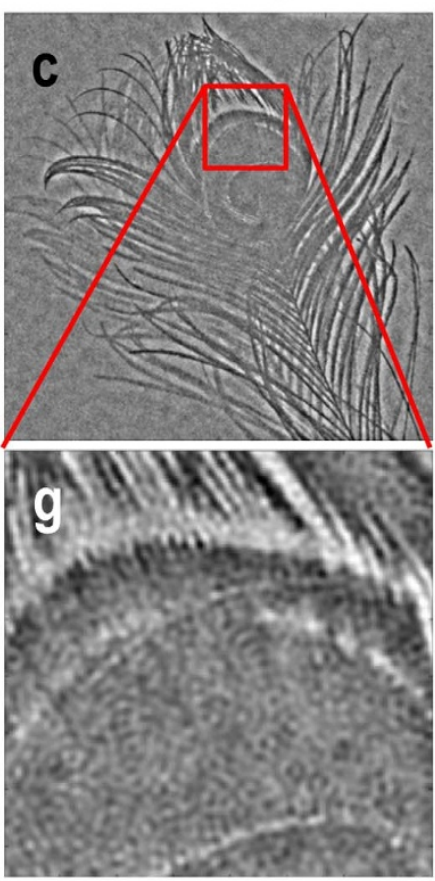

Hybrid image

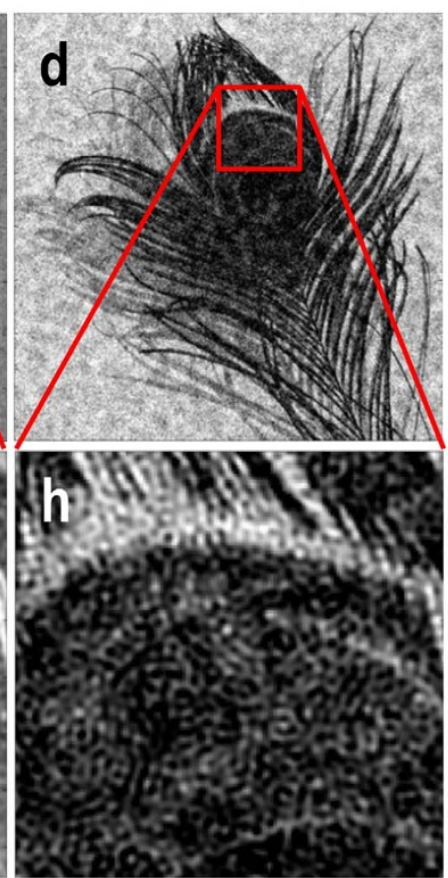

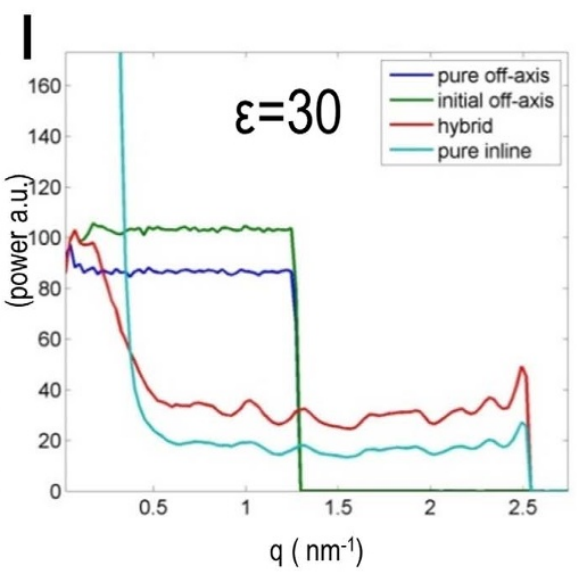

Figure 4 | Simulations: Comparison of noise and resolution of phase maps reconstructed from different simulated data sets with noise properties corresponding to equal total exposure times and electron doses. (a) The original, noise-free phase map $(0 \leq \phi(r) \leq 0.9)$ used for simulating off-axis and in-line holograms. The red square indicates the area from which Figs. (e)-(h) have been extracted, in each case from the phase map immediately above it. (b) Off-axis holography reconstruction for an exposure time of $0.4 \mathrm{~s}$, (c) In-line holography reconstruction from 7 equally long exposed images with a total exposure time of $0.4 \mathrm{~s}$. (d) Hybrid (off-axis + in-line) reconstruction. Exposure time for the initial off-axis hologram: $0.1 \mathrm{~s}$ and for the complete focal series: $0.3 \mathrm{~s}$. (i) Plot of the square root of the azimuthally averaged power spectrum of the difference between reconstruction and original, i.e.

$\sqrt{\text { mean }\left(\left|F T\left[\phi_{\text {rec }}-\phi_{\text {orig }}\right]\right|^{2}(q)\right)}$ for the cases (b), (c), and (d), as well as for the initial off-axis reconstruction used as a starting guess for the hybrid approach. The simulated data from which these reconstructions were performed are shown in Supplementary Fig. 5, along with a detailed description of the assumed acquisition parameters. (k) and (l), same as (i), but for elliptical illumination conditions.

holography alone, promising an improvement in the reliability of quantitative holography-based experiments that are aimed at mapping electric and magnetic fields, charge distributions and strain.

In simulations, where we can directly compare the reconstruction with the expected result, this comparison is more straightforward, and we can also quantify the standard deviation of the power spectra of actual and reconstructed phases, as shown in Figs. 4i, 4k, and $4 \mathrm{l}$. The increase in spatial resolution and simultaneous decrease in noise, despite equal total exposure times, becomes very obvious when comparing Figs. $4 \mathrm{~b}$ (magnified in $4 \mathrm{f}$ ) and $4 \mathrm{~d}$ (magnified in $4 \mathrm{~h}$ ).

The mean inner potential of the specimen was obtained as a demonstration of the capability of the method by dividing the measured phase by the local specimen thickness (Fig. 7b), which was measured from an energy-filtered TEM (EFTEM) thickness map and by a wavelength-dependent electron-matter interaction constant. The mean inner potential at the edge of the specimen, which consists of carbon, is found to be close to the theoretical value found in the literature ${ }^{39}$. The amplitude of the reconstructed wavefunction can also be used to obtain a thickness-independent mean inner potential image, as shown in Supplementary Fig. 2. The main error in determining the mean inner potential is the accuracy with which the local specimen thickness can be determined.

A further advantage of the hybrid method is its applicability to beam-sensitive specimens. Off-axis electron holography has high phase sensitivity at low spatial frequencies, requires a short exposure time and imparts a low total electron dose on the specimen since it is 

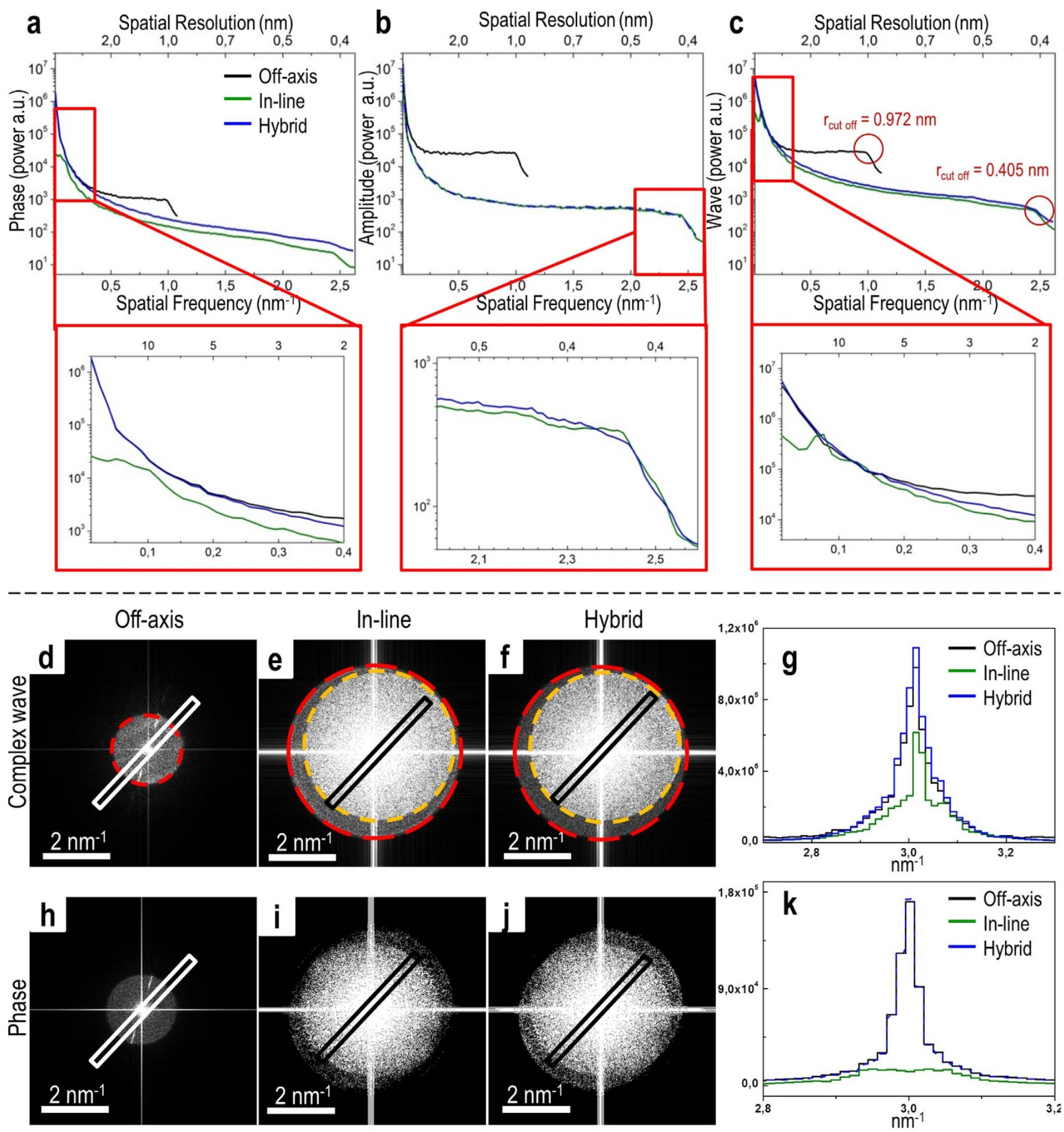

Figure 5 Information transfer: Information transfer of (a) phase, (b) amplitude and (c) complex wavefunction plotted as a function of spatial frequency for off-axis (black), in-line (green) and hybrid (blue) methods, with enlargements of selected regions shown below. The cut-off resolutions for the off-axis method $(0.972 \mathrm{~nm})$ and the in-line method $(0.405 \mathrm{~nm})$ are marked in $(\mathrm{c})$. (d-f) show power spectra of the complex wavefunction calculated for the (d) off-axis, (e) in-line and (f) hybrid methods. The shadow of the objective aperture used in the microscope is outlined in yellow in (e) and (f), while the red circle shows the cut-off frequency applied during reconstruction. The power spectra show how the low spatial frequency information that is missing in results obtained using in-line electron holography is recovered when using the hybrid approach. The horizontal and vertical lines in the power spectra are artifacts resulting from non-periodic boundaries of the images.

a single shot method. However, the exposure time needs to be increased to achieve high phase sensitivity at higher spatial resolution. Focal series acquisition schemes can be optimized for beamsensitive samples by first acquiring images at small defocus values (for retrieving high spatial frequency information which suffers first from potential beam damage) and only then recording images with large defocus values ${ }^{22}$. Partial spatial coherence of the illuminating electron beam results in strong damping of the fine details of images recorded for large over- or under- focus, making these images comparatively insensitive to small structural changes produced by electron irradiation. In this way, the data ideally become increasingly insensitive to beam damage with increasing electron dose. In the 

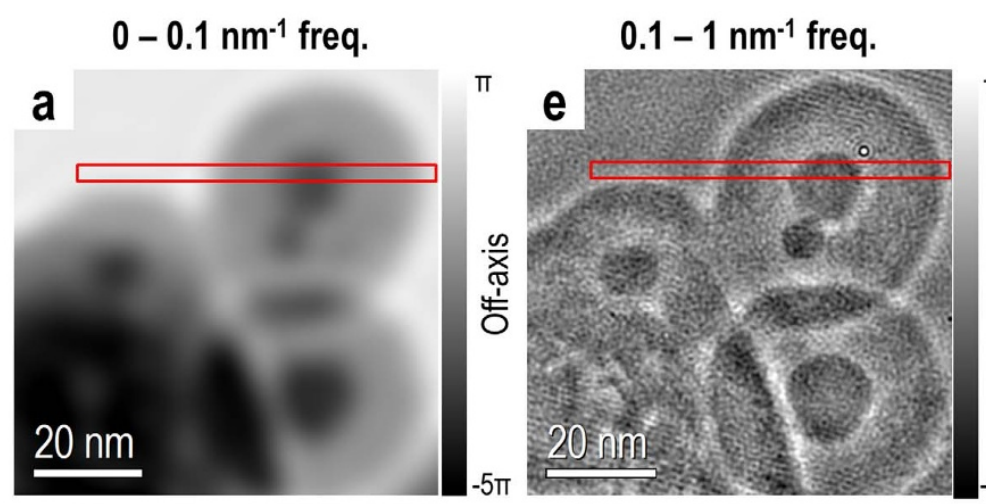

$0.1-1 \mathrm{~nm}^{-1}$ freq.

b

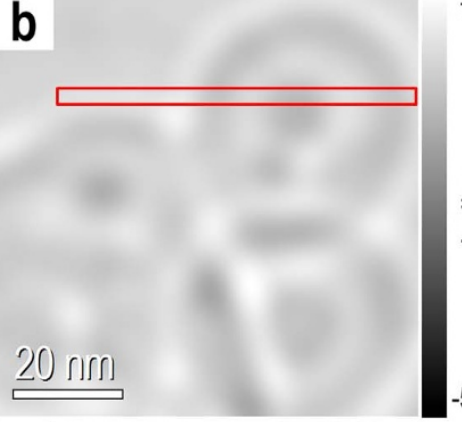

C

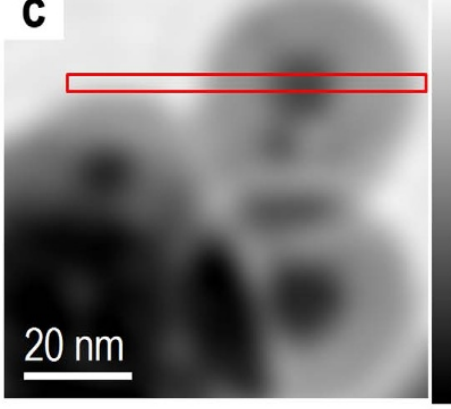

d

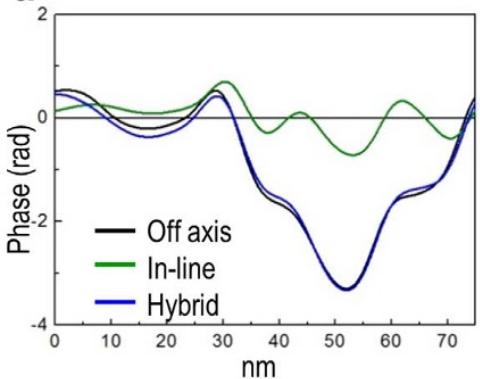

$\pi \quad f$

$-5 \pi$

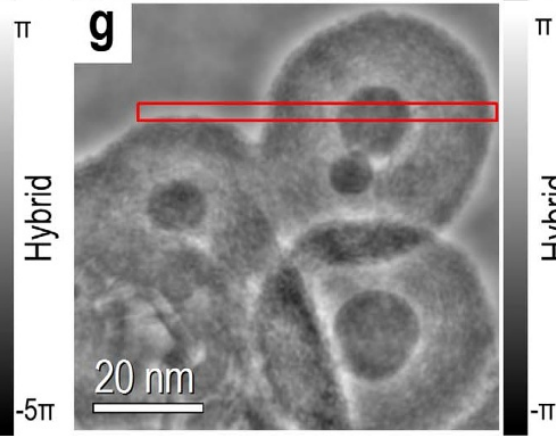

$-5 \pi$

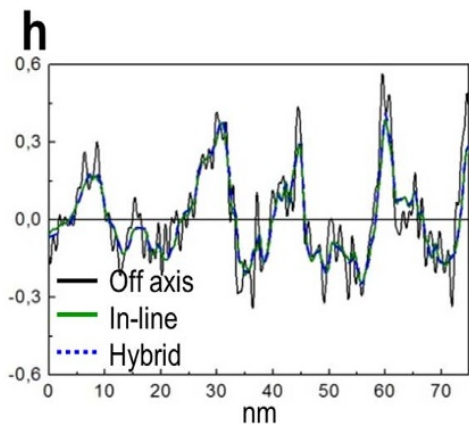

$1-2.5 \mathrm{~nm}^{-1}$ freq.

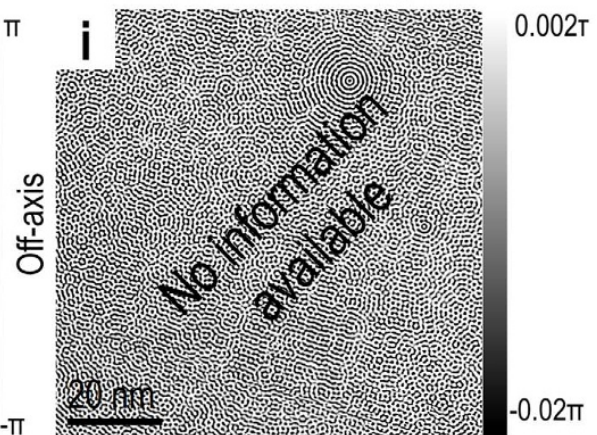

$\pi$

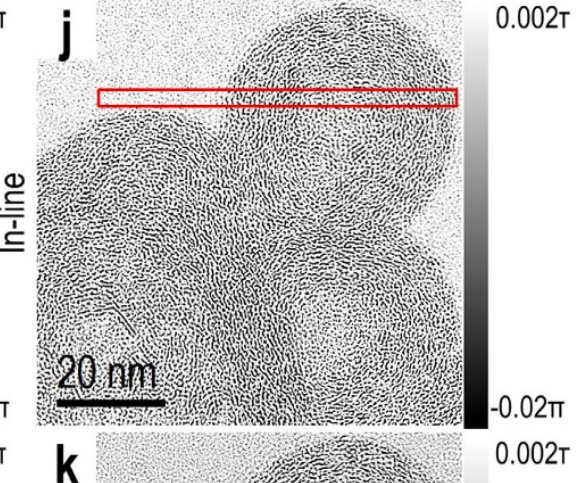

$20 \mathrm{~nm}$

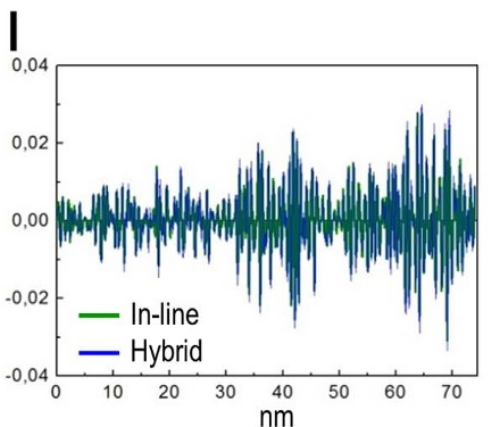

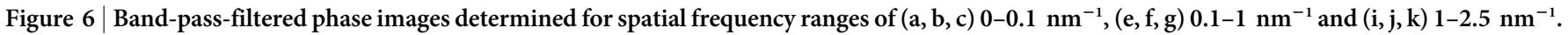
Top row: off-axis; middle row: in-line; bottom row: hybrid methods. Line profiles determined by projecting the intensity in the boxes marked in red are shown in (d), (h) and (i).

Table 1 | Noise levels (standard deviations) of reconstructed phase and amplitude measured in the vacuum region

\begin{tabular}{llllll|} 
& & \multicolumn{3}{c}{ Frequency range $\left(\mathrm{nm}^{-1}\right)$} \\
\cline { 3 - 6 } & Method & \multicolumn{1}{c}{$0-0.1$} & $0.1-1$ & $1-2.5$ & Full range \\
\hline Phase & Off-axis & 0.021 & 0.1 & - & 0.110 \\
& In-line & 0.006 (not reliable) & 0.018 & 0.005 & 0.017 (not completely reliable) \\
Amplitude & Hybrid & 0.006 & 0.012 & 0.006 & 0.026 \\
& Off-axis & 0.158 & 3.796 & - & 3.124 \\
& In-line & 0.008 & 0.069 & 0.096 & 0.114 \\
& Hybrid & 0.006 & 0.072 & 0.091 & 0.115 \\
\hline
\end{tabular}




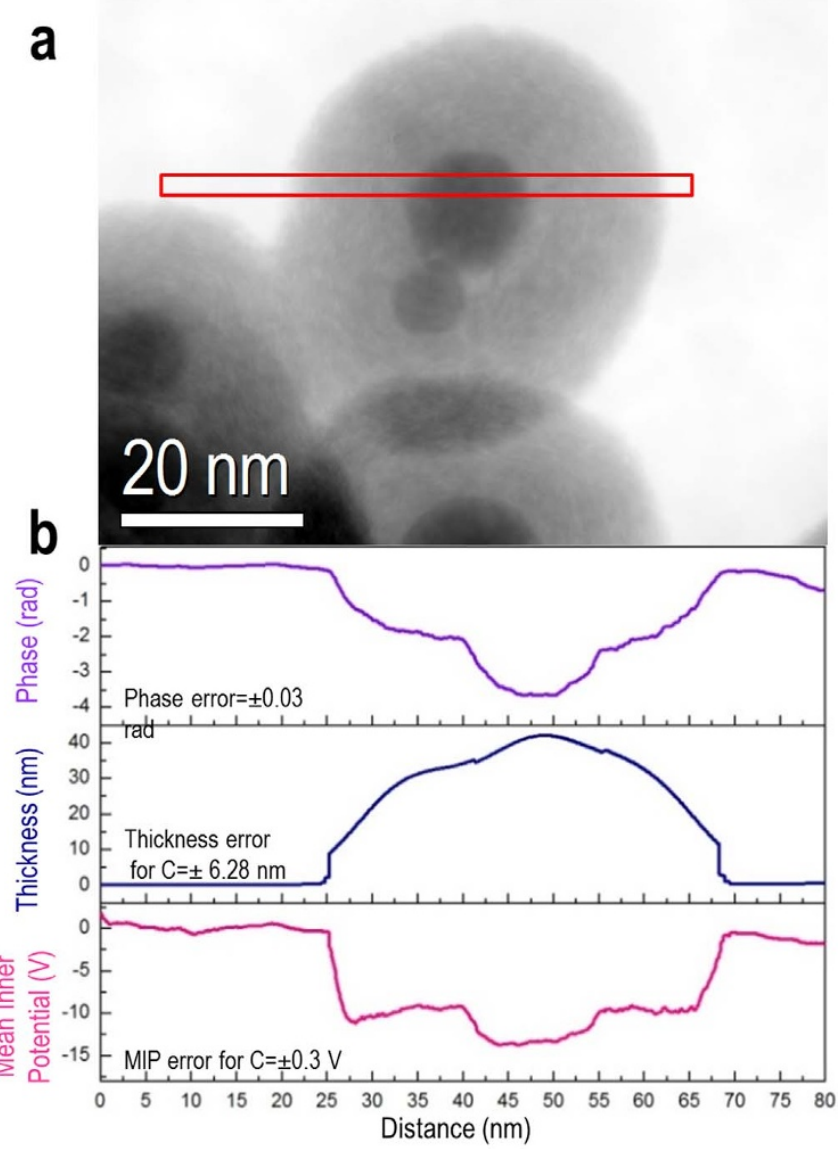

Figure $7 \mid$ Mean inner potential calculated from reconstructed phase image obtained using the hybrid method. (a) Original phase image; (b) phase, thickness and calculated mean inner potential profiles from the marked region shown in (a).

hybrid method, both the off-axis exposure time and the number of images in the focal series can be decreased to reduce the electron dose. Although beam damage was not an issue in the present work, Supplementary Fig. 3 shows that the hybrid method by including inline electron holograms recorded at only 3 different planes of focus can recover the phase with very low noise and high spatial resolution. However, the recovery of the wavefunction from 13 images is better, as can be seen from the correct recovery of the shadow of the objective aperture in Figure $5 \mathrm{f}$.

In summary, we have demonstrated that the major weaknesses of in-line and off-axis electron holography can be overcome by combining the two techniques, resulting in a hybrid method that can be used to reconstruct a complex electron wavefunction with high spatial resolution and low noise over all of the spatial frequencies that are collected during the experiment, with relaxed experimental requirements for instrumental stability and interference fringe spacing. In the example presented here, a full spatial frequency range was achieved, providing an improvement over the absence of low spatial frequencies when using in-line holography alone. Even though the hybrid technique adds an additional step to the experimental procedure and may very slightly increase the noise at very low spatial frequencies when compared to off-axis electron holography alone, the total acquisition time uses the electron dose more efficiently to recover more of the wavefunction. The same overall phase sensitivity and noise level cannot be achieved using off-axis or in-line electron holography alone, given the same electron source brightness and exposure time. The efficient use of electron dose realized by the hybrid technique offers great potential for applications to biological materials and high-resolution studies.

\section{Methods}

In off-axis electron holography, the electrostatic biprism attracts the spatially coherent electron wave function on either side of it towards the optic axis of the microscope, thereby introducing a relative wavevector $q_{c}$ between an object wave and a reference wave. The reference wave, which is usually part of the electron wavefunction that has not been scattered by the object, can often be regarded simply as a tilted plane wave. The Fourier transform of the resulting interference pattern, which corresponds to the sum of an object wave $\mathrm{e}^{2 \pi \mathrm{i} q_{c} \cdot r} \times \mathrm{A}(r) \cdot \mathrm{e}^{\mathrm{i} \phi(r)}$ and a reference wave $\pm \mathrm{e}^{-2 \Pi \mathrm{i} q_{c} \cdot r}$, is given by the expression ${ }^{13}$

$$
F T\left\{I_{\text {hol }}(\vec{r})\right\}=\left\{\begin{array}{cc}
F T\left\{1+A^{2}(\vec{r})\right\} & \text { central band } \\
+\mu F T\left\{A(\vec{r}) e^{i \phi(\vec{r})}\right\} \otimes \delta\left(\vec{q}-\overrightarrow{q_{c}}\right) & \text { sideband }+1 \\
+\mu F T\left\{A(\vec{r}) e^{i \phi(\vec{r})}\right\} \otimes \delta\left(\vec{q}+\overrightarrow{q_{c}}\right) & \text { sideband }-1
\end{array}\right.
$$

where $\boldsymbol{q}$ is the two-dimensional reciprocal space coordinate, $A(\boldsymbol{r})$ and $\phi(\boldsymbol{r})$ are the amplitude and phase of the object wavefunction and $\mu$ is the contrast of the holographic interference fringes. As shown in Fig. 1a, in order to achieve the necessarily very high spatial coherence perpendicular to the electrostatic biprism required for off-axis electron holography, the illumination is setup to be highly elliptical. The degree of ellipticity is defined by the number $\varepsilon$, which is simply the ratio of the illumination convergence angles for the short and long axis of the ellipse. If the shear $\mathbf{q}_{\mathbf{c}}$ is large enough, then the sidebands are separated from the central band in reciprocal space and a simple inverse Fourier transform of one of the sidebands yields the reconstructed wavefunction. Since only a relatively small part of the data is used for reconstruction, the resolution is at least 3-4 times lower than that of the recorded data set.

In non-linear in-line electron holography, the object wavefunction is its own reference wave, avoiding the need for an electrostatic biprism. The wavefunction can then potentially be reconstructed at the full image resolution. In this paper, we employ an iterative flux-preserving in-line electron holography reconstruction algorithm ${ }^{18}$, which minimizes the difference between defocused images (in-line holograms) simulated from the current estimate of the electron wavefunction at each iteration and the experimental measurement, taking into account incoherent aberrations (partial spatial and temporal coherence) and refining values of experimental parameters such as defocus $\Delta f$, the illumination convergence semi-angle $\alpha$, image registration and defocus-induced distortions, which are all only known approximately. The image intensity $I(\boldsymbol{r}, \Delta f, \varepsilon)$ of any member of the focal series at defocus $\Delta f$ and illumination ellipticity $\varepsilon$, from which the wave function $\Psi(r)$ is to be reconstructed is given by

$$
I(\vec{r}, \Delta f, \varepsilon)=F T^{-1}\left[F T\left[I_{\Delta}(\vec{r}, \Delta f)\right] \cdot E_{\text {spat }}(\vec{q}, \Delta f, \varepsilon) \cdot M T F(\vec{q})\right]
$$

where,

$$
\begin{aligned}
& I_{\Delta}(\vec{r}, \Delta f)=\left|F T^{-1}\left[F T[\Psi(\vec{r})] \cdot C T F(\vec{q}, \Delta f) \cdot E_{\text {temp }}(\vec{q}) \cdot H(\vec{q})\right]\right|^{2} \\
& \operatorname{CTF}(\vec{q}, \Delta f)=\exp \left(i \pi \lambda|\vec{q}|^{2}\left[\Delta f+0.5 \lambda^{2} C_{S}|\vec{q}|^{2}\right]\right) \\
& E_{\text {temp }}(\vec{q})=\exp \left(-\left[0.5 \pi \lambda \Delta_{f}|\vec{q}|^{2}\right]^{2}\right) \\
& H(\vec{q})= \begin{cases}1, & \lambda|\vec{q}| \leq \theta_{\max } \\
0, & \lambda|\vec{q}|>\theta_{\max }\end{cases} \\
& E_{\text {spat }}(\vec{q}, \Delta f, \varepsilon)=\exp \left(-\left[\pi \alpha \lambda \Delta \mathrm{f}\left(q_{x}^{2}+\varepsilon q_{y}^{2}\right)\right]^{2}\right) .
\end{aligned}
$$

In Eq. 2, the flux-preserving approximation ${ }^{18}$ has been adopted, which, in case of negligible effect of the spherical aberration $C_{\mathrm{s}}$ on the spatial coherence envelope $\mathrm{E}_{\text {spat }}(\boldsymbol{q})(\mathrm{Eq} .7)$, accounts more accurately for the intensity distribution in in-line holograms, than the more widely used quasi-coherent approximation. In the above formulae the coherent transfer function $\operatorname{CTF}(\boldsymbol{q})$ (Eq. 4) has (for readability reasons) been limited to include only the effect of defocus $(\Delta f)$ and spherical aberration $\left(C_{\mathrm{s}}\right)$, but can easily be extended to include any other coherent aberration coefficient as well. In contrast to earlier work, the ellipticity $\varepsilon$ of the illumination is explicitly included in the envelope function accounting for partial spatial coherence $\left(\mathrm{E}_{\text {spat }}(\boldsymbol{q})\right)$ which here assumes the direction of high spatial coherence (characterized by the semiconvergence angle $\alpha$ ), i.e. the direction normal to the biprism in an off-axis electron holography experiment, to lie along the $\mathrm{x}$-axis. Furthermore, an objective aperture $\left(\mathrm{H}(\boldsymbol{q})\right.$ ) (Eq. 6) admitting only scattering angles less than $\theta_{\max }$ and a partial temporal coherence envelope $\left(\mathrm{E}_{\mathrm{temp}}(q)\right)$ (Eq. 5) depending on the focal spread $\Delta_{\mathrm{f}}$ are considered.

Nano-catalyst particles with a core-shell structure, consisting of carbon nanospheres with iron cores, were selected as a test material to assess the limits of the method. The core-shell particles have fine feature sizes between $\sim 0.5$ and $0.8 \mathrm{~nm}$ since the carbon phase is only partially crystalline. The carbon layers are buckled, and display clear phase contrast. This sample therefore works very well as a test object for assessing the spatial resolution of the method. In order to combine the two methods, an off-axis hologram and a focal series were recorded from the same area using an FEI 
Titan 80-300 TEM equipped with two electron biprisms and a Gatan imaging filter with a $2048 \times 2048$ pixel charge-coupled device $(\mathrm{CCD})$ camera. The experiment was carried out at an acceleration voltage of $300 \mathrm{kV}$. For this experiment both off-axis and in-line acquisitions were done using round illumination conditions having inserted a $30 \mu \mathrm{m}$ objective aperture. First the off-axis electron hologram was acquired using a biprism voltage of $89.4 \mathrm{~V}(0.38 \mathrm{~nm}$ fringe spacing) to obtain an optimum field of view and resolution and a $20 \mathrm{~s}$ exposure time. Then, the biprism was turned off and retracted from the beam. The sample was shifted to bring the same area of interest back on the detector and a focal series was acquired from the same area using the FWRWtools ${ }^{40}$ plugin, applying linear defocus increments with a $90 \mathrm{~nm}$ defocus step size. The illumination conditions were not changed between the off-axis and in-line electron holography data acquisitions (Fig. 1c). At each defocus, an image was acquired using a 3 s exposure time. The objective lens was used for changing the focus, following assumptions that are explained elsewhere ${ }^{18}$. For both off-axis and inline electron holography, zero-loss filtering, employing a $10 \mathrm{eV}$ energy-selecting slit was used, in order to reduce the contribution of inelastically scattered electrons.

Reconstruction of the off-axis electron hologram was performed using the HolograFREE ${ }^{41}$ software. For in-line and hybrid reconstruction, a flux preserving non-linear in-line holography reconstruction algorithm ${ }^{18}$ was used. This method takes into account the modulation transfer function (MTF) of the CCD camera (whose effect is shown in Supplementary Fig. 4), as well as partial spatial coherence and defocus-induced image distortions. The algorithm also refines experimental parameters such as defocus and the illumination convergence angle. In order to combine the two methods, the same region of interest that was selected for in-line electron holography was aligned with the amplitude images obtained from the offaxis reconstruction. Then, the in-line reconstruction algorithm was re-run, starting from the off-axis amplitude and (unwrapped) phase, refining the imaging parameters that were fitted during the first in-line reconstruction. Since the phase and amplitude that were obtained from the off-axis data were also used for an initial guess using the hybrid method, the same illumination conditions were assumed for the off-axis and in-line data. The thickness measurement required for determining the mean inner potential was obtained by EFTEM thickness mapping, with mean free paths calculated using David Mitchell's mean free path estimator script ${ }^{42}$. In the region at the center of the particle, where both iron $(\mathrm{Fe})$ and carbon $(\mathrm{C})$ are present, a mean free path of $183.3 \mathrm{~nm}$ was assumed. This value was calculated according to the volume ratio of $\mathrm{Fe}$ to $\mathrm{C}$, for which the $\mathrm{Fe}$ core was assumed to be spherical with a radius of $7.5 \mathrm{~nm}$ and the particle radius was assumed to have a measured value of $22.5 \mathrm{~nm}$. For the $\mathrm{C}$ shell region, a mean free path of $188.3 \mathrm{~nm}$ was used.

1. Gabor, D. A New Microscopic Principle. Nature 161, 777-778 (1948).

2. Lundqvist, S. [Holography]. Nobel Lectures in Physics 1971-1980, World Scientific, Singapore, 1992.

3. Orchowski, A., Rau, W. D. \& Lichte, H. Electron Holography Surmounts Resolution Limit of Electron Microscopy. Phys. Rev. Lett. 74, 399-402 (1995).

4. Rau, W. D., Schwander, P., Baumann, F. H., Höppner, W. \& Ourmazd, A. TwoDimensional Mapping of the Electrostatic Potential in Transistors by Electron Holography. Phys. Rev. Lett. 82, 2614-2617 (1999).

5. Loudon, J. C., Mathur, N. D. \& Midgley, P. A. Charge-ordered ferromagnetic phase in La0.5Ca0.5MnO3. Nature 420, 797-800 (2002).

6. He, K., Ma, F.-X., Xu, C.-Y. \& Cumings, J. Mapping magnetic fields of Fe3O4 nanosphere assemblies by electron holography. J. Appl. Phys. 113, 17B528 (2013).

7. Hÿtch, M., Houdellier, F., Hüe, F. \& Snoeck, E. Nanoscale holographic interferometry for strain measurements in electronic devices. Nature $\mathbf{4 5 3}$ 1086-1089 (2008)

8. Koch, C. T., Özdöl, V. B. \& Aken, P. A. van. An efficient, simple, and precise way to map strain with nanometer resolution in semiconductor devices. Appl. Phys. Lett. 96, 091901 (2010).

9. Twitchett-Harrison, A. C., Yates, T. J. V., Newcomb, S. B., Dunin-Borkowski, R. E. \& Midgley, P. A. High-Resolution Three-Dimensional Mapping of Semiconductor Dopant Potentials. Nano Lett. 7, 2020-2023 (2007).

10. Simon, P. et al. Electron holography of biological samples. Micron 39, 229-256 (2008).

11. Cowley, J. M. Twenty forms of electron holography. Ultramicroscopy 41, 335-348 (1992).

12. Möllenstedt, G. \& Düker, H. Fresnelscher Interferenzversuch mit einem Biprisma für Elektronenwellen. Naturwissenschaften 42, 41-41 (1955).

13. Lehmann, M. \& Lichte, H. Tutorial on Off-Axis Electron Holography. Microsc. Microanal. 8, 447-466 (2002).

14. Möllenstedt, G. \& Wahl, H. Elektronenholographie und Rekonstruktion mit Laserlicht. Naturwissenschaften 55, 340-341 (1968).

15. Haine, M. E. \& Dyson, J. A Modification to Gabor's Proposed Diffraction Microscope. Nature 166, 315-316 (1950).

16. Kirkland, E. J. Improved high resolution image processing of bright field electron micrographs: I. Theory. Ultramicroscopy 15, 151-172 (1984).

17. Coene, W. M. J., Thust, A., Op de Beeck, M. \& Van Dyck, D. Maximum-likelihood method for focus-variation image reconstruction in high resolution transmission electron microscopy. Ultramicroscopy 64, 109-135 (1996).

18. Koch, C. T. A flux-preserving non-linear inline holography reconstruction algorithm for partially coherent electrons. Ultramicroscopy 108, 141-150 (2008).

19. Reed Teague, M. Deterministic phase retrieval: a Green's function solution. J. Opt. Soc. Am. 73, 1434-1441 (1983).
20. Kirkland, A. I., Saxton, W. O., Chau, K.-L., Tsuno, K. \& Kawasaki, M. Superresolution by aperture synthesis: tilt series reconstruction in CTEM. Ultramicroscopy 57, 355-374 (1995)

21. Haigh, S. J., Sawada, H. \& Kirkland, A. I. Optimal tilt magnitude determination for aberration-corrected super resolution exit wave function reconstruction. Philos. Transact. A Math. Phys. Eng. Sci. 367, 3755-3771 (2009).

22. Koch, C. T. Towards full-resolution inline electron holography. Micron 69, 69-75 (2014).

23. Haigh, S. J., Jiang, B., Alloyeau, D., Kisielowski, C. \& Kirkland, A. I. Recording low and high spatial frequencies in exit wave reconstructions. Ultramicroscopy 133, 26-34 (2013).

24. Dunin-Borkowski, R. E. The development of Fresnel contrast analysis, and the interpretation of mean inner potential profiles at interfaces. Ultramicroscopy $\mathbf{8 3}$, 193-216 (2000).

25. Twitchett, A. C., Dunin-Borkowski, R. E. \& Midgley, P. A. Comparison of off-axis and in-line electron holography as quantitative dopant-profiling techniques. Philos. Mag. 86, 5805-5823 (2006).

26. Koch, C. T. \& Lubk, A. Off-axis and inline electron holography: A quantitative comparison. Ultramicroscopy 110, 460-471 (2010).

27. Latychevskaia, T., Formanek, P., Koch, C. T. \& Lubk, A. Off-axis and inline electron holography: Experimental comparison. Ultramicroscopy 110, 472-482 (2010).

28. Koch, C. T. Determination of grain boundary potentials in ceramics: Combining impedance spectroscopy and inline electron holography. Int. J. Mater. Res. 101, 43-49 (2010)

29. Geilhufe, J. et al. Monolithic focused reference beam X-ray holography. Nat. Commun. 5 (2014)

30. Ruhlandt, A., Krenkel, M., Bartels, M. \& Salditt, T. Three-dimensional phase retrieval in propagation-based phase-contrast imaging. Phys. Rev. A 89, 033847 (2014).

31. Rosenhahn, A. et al. Digital in-line soft x-ray holography with element contrast. J. Opt. Soc. Am. A Opt. Image Sci. Vis. 25, 416-422 (2008).

32. Wachulak, P. W., Bartels, R. A., Marconi, M. C., Menoni, C. S. \& Rocca, J. J. Table Top Extreme Ultraviolet Holography. in Conf. Lasers Electro-Opt. Electron. Laser Sci. Conf. Photonic Appl. Syst. Technol. CMX3 (Optical Society of America, 2007). at $<$ http://www.opticsinfobase.org/abstract.cfm?URI =CLEO-2007-CMX3 $>$.

33. Baars, J. W. M., Lucas, R., Mangum, J. G. \& Lopez-Perez, J. A. Near-Field Radio Holography of Large Reflector Antennas. ArXiv07104244 Astro-Ph at http://arxiv. org/abs/0710.4244 (2007)

34. Khare, K., Ali, P. T. S. \& Joseph, J. Single shot high resolution digital holography. Opt. Express 21, 2581-2591 (2013).

35. Kemper, B., Langehanenberg, P. \& von Bally, G. Digital Holographic Microscopy. Opt. Photonik 2, 41-44 (2007).

36. Ophus, C. \& Ewalds, T. Guidelines for quantitative reconstruction of complex exit waves in HRTEM. Ultramicroscopy 113, 88-95 (2012).

37. Joy, D. C. SMART-a program to measure SEM resolution and imaging performance. J. Microsc. 208, 24-34 (2002).

38. Yi, J. et al. High-resolution hard- $\mathrm{x}$-ray microscopy using second-order zone-plate diffraction. J. Phys. Appl. Phys. 44, 232001 (2011).

39. Schowalter, M., Titantah, J. T., Lamoen, D. \& Kruse, P. Ab initio computation of the mean inner Coulomb potential of amorphous carbon structures. Appl. Phys. Lett. 86, 112102-112102-3 (2005).

40. Koch, C. T. Useful Plugins and Scripts for DigitalMicrograph | ELIM: Electron and Ion Microscopy. at http://elim.physik.uni-ulm.de/?page_id=564, Date of access: 29/09/14.

41. Bonevich, J. US Department of Commerce, N. Electron holography. at http:// www.nist.gov/mml/msed/functional_nanostructure/electron_holography.cfm, Date of access: 29/09/14.

42. Mitchell, D. Mean Free Path Estimator at http://portal.tugraz.at/portal/page/ portal/felmi/DM-Script/DM-Script-Database, Date of access: 29/09/14.

\section{Acknowledgments}

The authors are grateful to John Bonevich for offering free public use of the HolograFREE off-axis electron holography reconstruction software. C.T.K. thanks the Carl Zeiss Foundation and the German Research Foundation (DFG, grant No KO 2911/7-1) for financial support. R.D.B. thanks a European Research Council Advanced Grant for financial support. The research leading to these results received funding from the European Union Seventh Framework Programme [FP7/2007-2013] under grant agreement No312483 (ESTEEM2). The authors are also grateful to N.Y. Jin-Phillipp for providing the samples examined in the present study also to Alison F. Mark for help on the manuscript.

\section{Author contributions}

C.O.K. made substantial contributions to the conception of the experiment, design, acquisition of data, analysis, and interpretation and wrote the manuscript; C.B.B. supervised and helped with off-axis electron holography and made revisions to the paper; R.D.B. supervised the off-axis electron holography and made revisions to the paper; P.A.A. supervised the project and advised on conceptual design; C.T.K. supplied the in-line electron holography reconstruction algorithm and simulations, supervised the overall 
study, helped to draft the article and revised the manuscript for important intellectual content.

\section{Additional information}

Supplementary information accompanies this paper at http://www.nature.com/

scientificreports

Competing financial interests: The authors declare no competing financial interests.

How to cite this article: Ozsoy-Keskinbora, C., Boothroyd, C.B., Dunin-Borkowski, R.E., van Aken, P.A. \& Koch, C.T. Hybridization approach to in-line and off-axis (electron) holography for superior resolution and phase sensitivity. Sci. Rep. 4, 7020; DOI:10.1038/ srep07020 (2014)

(i) $\Theta$ This work is licensed under a Creative Commons Attribution-NonCommercialNoDerivs 4.0 International License. The images or other third party material in this article are included in the article's Creative Commons license, unless indicated otherwise in the credit line; if the material is not included under the Creative Commons license, users will need to obtain permission from the license holder in order to reproduce the material. To view a copy of this license, visit http:// creativecommons.org/licenses/by-nc-nd/4.0/ 